\title{
CONTROLE ESTATÍSTICO APLICADO AO PROCESSO DE COLHEITA MECANIZADA DE CANA-DE-AÇÚCAR
}

\author{
ROUVERSON P. DA SILVA ${ }^{1}$, CAIO F. CORRÊA ${ }^{2}$, JORGE W. CORTEZ ${ }^{3}$, \\ CARLOS E. A. FURLANI ${ }^{4}$
}

\begin{abstract}
RESUMO: O avanço da mecanização na colheita da cana-de-açúcar (Saccharum spp.) proporcionou o uso de novas tecnologias e ganho em produtividade para a cultura. O controle da qualidade do processo de colheita da cana-de-açúcar é fundamental para reduzir as perdas. Este trabalho teve o objetivo de avaliar as perdas na colheita mecanizada de cana-de-açúcar, utilizandoas como indicadores de qualidade do processo de colheita. Os dados foram coletados em duas propriedades próximas a Jaboticabal - SP, com a variedade SP80-3280, em $3^{\mathrm{O}}$ e $4^{\mathrm{o}}$ cortes. Caracterizou-se o porte do canavial e, após a colheita, demarcou-se área de 1,5 ha, sendo demarcados 25 pontos, espaçados de $12 \times 50 \mathrm{~m}$, quantificando-se as perdas visíveis. Posteriormente, foi aplicado o controle estatístico do processo pela média, que consta de três vezes o desvio-padrão para mais ou para menos, sendo esses os limites superior e inferior de controle, respectivamente. A média das perdas de pedaço solto foi estatisticamente maior do que as médias de perdas em pedaço fixo, cana inteira, cana-ponta e toco. A ocorrência de perdas em rebolo estilhaçado foi menor para o $4^{\underline{0}}$ corte em relação ao $3^{-0}$ corte, enquanto as perdas em pedaço fixo e toco foram menores no $3^{\underline{0}}$ corte, comparadas às perdas no $4^{\underline{0}}$ corte. Em cada corte, as médias para as perdas totais estiveram próximas dos valores encontrados na bibliografia. Pedaço solto foi a variável de perdas visíveis com maior percentagem de ocorrência. As perdas demonstraram que a colheita mecanizada não se encontra sob controle estatístico de processo.
\end{abstract}

PALAVRAS-CHAVE: perdas visíveis, colhedora de cana-de-açúcar, controle de qualidade.

\section{STATISTICAL CONTROL APPLIED IN THE PROCESS OF MECHANICAL SUGAR CANE HARVEST}

\begin{abstract}
The dvances in sugar cane mechanized harvest provided the management of new technologies and productivity gain to the crop. The control of quality process of sugar cane harvest is essential to reduce losses. However, studies regarding the subject are still scarce. This study aimed to evaluate the losses in the mechanized harvest of sugar cane, using them as markers of harvest quality process. The data was collected in two properties near Jaboticabal - SP, Brazil, on SP80-3280 variety, in $3^{\text {rd }}$ and $4^{\text {th }}$ cuts. The sugar cane plantation port was characterized, and, after the harvest, an area of 1.5 ha was marked, being 25 points, with row spacing of $12 \times 50 \mathrm{~m}$, quantifying the visible losses. Later, the statistical process quality was applied by the average, which is three times higher or lower than the standard deviation, being these control limits superior and inferior, respectively. The loss average of untied piece was statistically higher than the loss average of fixed piece, whole cane, top and stubbles. The occurrence of losses of splinted stalks was lower for the fourth cut in relation to the third curt, whereas the losses of fixed piece and stubbles were lower in the third cut when compared to the losses in the fourth cut. In each cut, the averages of total losses were similar to the values found in bibliography. Untied piece was the variable of visible losses with higher percentage of occurrence. The losses showed that the mechanized harvest is not under the statistical process control.
\end{abstract}

KEYWORDS: visible losses, sugar cane harvest machine, quality control.

\footnotetext{
${ }^{1}$ Eng ${ }^{\circ}$ Agrícola, Prof. Dr., Departamento de Engenharia Rural, UNESP, Jaboticabal - SP, Fone: (0xx16) 3209.2637, rouverson@fcav.unesp.br

${ }^{2}$ Eng $^{\underline{0}}$ Agrônomo, Cosan S.A. Indústria e Comércio, Fazenda da Serra, Ibaté - SP. Fone: (0XX16) 3343.1331, Caio.Correa@cosan.com.br

${ }^{3}$ Eng ${ }^{0}$ Agrônomo, Professor Assistente, UNIVASF, Câmpus Juazeiro - BA, Fone: (0XX74) 3614.1934, jorge.cortez@yahoo.com.br

${ }^{4}$ Eng ${ }^{\mathrm{O}}$ Agrônomo, Prof. Adjunto, Departamento de Engenharia Rural, UNESP, Jaboticabal - SP, Fone: (0xx16) 3209.2637, furlani@fcav.unesp.br

Recebido pelo Conselho Editorial em: 17-7-2007
}

Aprovado pelo Conselho Editorial em: 5-4-2008 


\section{INTRODUÇÃO}

A cana-de-açúcar (Saccharum spp.) está entre as culturas mais importantes do agronegócio brasileiro. O Brasil é o maior produtor mundial de açúcar e de álcool de cana-de-açúcar e destaca-se também como o maior exportador, sendo o Estado de São Paulo o maior produtor nacional.

As operações de colheita de cana-de-açúcar foram classificadas por RIPOLI (1996) em três subsistemas distintos: manual, semimecanizado e mecanizado. Tal classificação deve-se ao fato da existência de um sistema global que envolve o corte e o carregamento, o transporte e a recepção da matéria-prima. O subsistema mecanizado diferencia-se por as operações de corte, carregamento e transporte ocorrerem exclusivamente por meio de máquinas.

De acordo com medições feitas pelo CTC (Centro de Tecnologia Canavieira), 10\% da matéria-prima colhida é perdida no campo quando o corte é mecanizado, representando prejuízo da ordem de US\$ 450 milhões por ano (MAGALHÃES et al., 2006).

No corte mecanizado de cana-de-açúcar, os cortadores de base exercem influência direta nas perdas e na contaminação da matéria-prima com o solo, bem como nos possíveis danos causados à soqueira. VOLPATO et al. (2002) estudaram o funcionamento dos cortadores de base e procuraram estabelecer critérios para a otimização de um cortador de base-flutuante para seguimento do perfil de solo em colhedoras de cana-de-açúcar. Os resultados finais foram satisfatórios, e o mecanismo foi submetido a processo de otimização computacional, sem validação experimental, mostrando-se passível de ajustes, visto que houve melhoras no desempenho após as modificações.

Colmos e/ou frações deixados no campo também são evidentes no sistema mecanizado da colheita de cana-de-açúcar e são classificados como perdas visíveis. O aumento da velocidade de saída de ar dos extratores das colhedoras é uma proposta para reduzir os índices de matéria estranha, contudo pode aumentar as perdas visíveis em níveis inaceitáveis economicamente. Alterando-se a velocidade do extrator primário e monitorando com sensor (piezelétrico) desenvolvido especialmente para esse fim, NEVES et al. (2004) obtiveram resultados mostrando que a eficiência de limpeza do extrator primário (cerca de $85 \%$ ), as perdas de cana-de-açúcar em geral (entre $2,5 \%$ e 5,7\%) e a velocidade do ventilador estão diretamente relacionadas. As perdas (totais) e, principalmente lascas (entre $0,9 \%$ e 3,9\%), apontaram diferenças significativas entre os tratamentos estudados.

Ao avaliarem as perdas na colheita de cana-de-açúcar e a demanda de potência de uma colhedora de cana inteira, GARCIA et al. (2006) verificaram que o equipamento utilizado apresentou números próximos a 7,7\% de perdas na colheita de cana queimada.

CARVALHO FILHO (2000), estudando o desempenho operacional e econômico da colheita mecanizada de cana sem queima prévia, concluiu que a variável velocidade de deslocamento teve influência no estudo, assim como a eficiência do sistema de colheita. A velocidade de colheita de $5,4 \mathrm{~km} \mathrm{~h}^{-1}$ apresentou o melhor desempenho econômico, menores perdas no campo e menor índice de matéria estranha.

A busca e a exigência por produtos de excelência em qualidade, aliados a preços acessíveis, são o ponto fundamental para os consumidores no momento de adquirir uma mercadoria. Não muito longe disso, estão empresas e indústrias que compram matérias-primas e as beneficiam, buscando atender às exigências dos consumidores. $O$ fato é que a matéria-prima deve sair de sua origem, no caso da cana-de-açúcar, o campo, com o máximo de qualidade possível. Para FERNANDES et al. (2000), por localizarem-se no início do processo de produção, as operações agrícolas são de fundamental importância para a melhoria de todo o processo produtivo, uma vez que cada operação influi diretamente sobre a operação subseqüente, conseqüentemente, no desenvolvimento da planta.

O Controle Estatístico de Processo (CEP) tem como objetivo detectar rapidamente alterações dos parâmetros de determinados processos para que os problemas possam ser corrigidos antes que muitos itens não-conformes sejam produzidos (MINGOTI \& FIDELIS, 2001). 
De acordo com FERNANDES et al. (2000), sob o ponto de vista econômico, qualidade é a produção de serviços e de produtos a custos compatíveis com a atividade, com a obtenção de lucros para o produtor e que satisfaçam às necessidades dos consumidores. Sob o ponto de vista agronômico, qualidade é a realização das operações agrícolas ou a obtenção de produtos que estejam adequados às especificações ou a padrões agronômicos recomendados.

O início da produção em empresas e indústrias começa no término da produção de matériaprima que, no caso da cana-de-açúcar, é representado pela colheita. Esse é o ponto fundamental para avaliar a qualidade do material que será processado, por isso estudos avaliando as perdas na colheita são importantes. Segundo BONILLA (1995), algumas ferramentas destacam-se no Controle Estatístico de Processo (CEP), sendo elas: histogramas, cartas de controle por variáveis, medidas de tendência central (média aritmética, mediana e moda), medidas de dispersão (amplitude, desvio-padrão e coeficiente de variação) e medidas de assimetria e de curtose. Diversos autores utilizam a carta de controle em seus experimentos, possibilitando observar variações ou oscilações em operações agrícolas que estejam fora dos padrões especificados para o processo. A carta de controle é composta por uma linha média e outras duas linhas (superior e inferior), que representam os limites de controle e os valores característicos do processo. Os limites de controle são estimados pelo valor médio, somado ou subtraído a três vezes o desvio-padrão. Quando todos os pontos do gráfico se localizam entre os limites de controle, considera-se que o processo está sob controle. Quando, no mínimo, um ponto se localiza fora desses limites, considera-se que o processo está fora de controle.

TRINDADE et al. (2000) sugerem que a elaboração das cartas básicas de controle pode ser por variáveis ou por atributos. Nas cartas básicas por variáveis, obtém-se a variação de modo quantitativo, podendo ser subdivididas em cartas de controle pela média, pela amplitude e pelo desvio-padrão, e cartas de dispersão do desvio-padrão e da amplitude. No caso das cartas de controle por atributos, a variação é obtida de modo qualitativo, podendo ser subdivididas em cartas da fração defeituosa e cartas do número total de defeitos por unidade.

O uso do CEP, a princípio, foi desenvolvido e utilizado por empresas buscando melhoria de qualidade e de produtividade. Bons resultados e a possibilidade de utilizar-se dessas ferramentas na agropecuária abriram caminhos para que estudos fossem realizados, principalmente em operações mecanizadas. MILAN \& FERNANDES (2002) testaram a qualidade das operações de preparo do solo por controle estatístico de processo e concluíram que a utilização do controle na operação de escarificação proporcionou redução da variabilidade de dados de profundidade de trabalho média em $38,4 \%$ e aumento de $45,0 \%$ de pontos amostrados com profundidade igual à estipulada pelos técnicos (entre 0,20 e 0,25 m). Na gradagem, houve redução de 9,8\% dos dados médios de tamanho de torrões e aumento de $75,0 \%$ de torrões aceitáveis. A implantação do controle nas operações foi viável e adequada ao sistema de produção em questão.

Conforme DODSON (1998), sob o ponto de vista agronômico, a qualidade pode ser definida como a realização de operações agrícolas ou a obtenção de produtos que estão dentro de especificações ou de determinados padrões agronômicos recomendados. $\mathrm{O}$ autor constatou, ainda, que fatores relacionados com a qualidade não são avaliados adequadamente, dificultando a verificação de sua influência no rendimento financeiro de produção. O CEP foi utilizado pelo autor para identificar pontos que apresentassem problemas e que não se adequassem às especificações agronômicas, na operação de semeadura de milho. Assim, DODSON (1998) procurou comprovar que a qualidade operacional exerce influência no rendimento final, uma vez que indicadores de qualidade escolhidos se mostraram adequados para inferir se o resultado da operação alcançou ou não a qualidade desejada. A sugestão para abrir nova linha de pesquisa, em fatores ligados à qualidade de implantação e condução de lavouras, é válida, pois os resultados obtidos foram satisfatórios.

Diante disso, este trabalho teve por objetivo avaliar as perdas na colheita mecanizada de canade-açúcar por meio do controle estatístico de processo, bem como verificar a qualidade da operação. 


\section{MATERIAL E MÉTODOS}

A colheita foi conduzida na Fazenda Bela Vista, Jaboticabal - SP, localizada nas coordenadas geográficas: latitude $21^{\circ} 18^{\prime} \mathrm{S}$ e longitude $48^{\circ} 11^{\prime} \mathrm{W}$, com altitude média de $680 \mathrm{~m}$, declividade média de $8 \%$, e na Fazenda Morumbi, Guariba - SP, cujas coordenadas geográficas são: latitude $21^{\circ} 25^{\prime}$ 'S e longitude $48^{\circ} 07^{\prime} \mathrm{W}$, com altitude média de $530 \mathrm{~m}$ e declividade média de $6 \%$. As propriedades encontram-se distantes $20 \mathrm{~km}$ uma da outra e apresentam clima Cwa (subtropical), de acordo com a classificação de Köeppen.

Foram amostradas duas áreas de 1,5 ha cada, sendo uma na Fazenda Bela Vista e outra na Fazenda Morumbi, de dimensões $60 \times 250 \mathrm{~m}$ cada, nas quais foram demarcados 25 pontos, espaçados de $12 \times 50 \mathrm{~m}$, formando malhas retangulares. A variedade de cana-de-açúcar cultivada nas propriedades foi a SP80-3280, desenvolvida pela Copersucar. Na Fazenda Bela Vista, a canade-açúcar avaliada estava em seu $3^{\circ}$ corte, enquanto na Fazenda Morumbi, estava no $4^{\circ}$ corte. Os pontos amostrados foram identificados com auxílio de um aparelho de GPS da marca Garmin, modelo GPSII (1 a $10 \mathrm{~m}$ de precisão). As coordenadas foram registradas em UTM, cujo sistema referencial de localização terrestre é baseado em coordenadas métricas definidas para cada uma das 60 zonas UTM; neste caso, a área encontrava-se na zona 22 (UNB, 2006).

Realizou-se a colheita da cana-de-açúcar crua com uma colhedora CASE-IH, modelo A7700, fabricada em 2002, equipada com motor Cummins, cuja potência nominal é de $184 \mathrm{~kW}(250 \mathrm{cv})$. Essa colhedora possui rodados de esteiras com bitola de $1,88 \mathrm{~m}$. A velocidade média de deslocamento da colhedora foi de $5,6 \mathrm{~km} \mathrm{~h}^{-1}$ e $7,5 \mathrm{~km} \mathrm{~h}^{-1}$ para o $3^{-}$e $4^{\underline{0}}$ cortes, respectivamente.

Para realizar a caracterização do porte dos canaviais avaliados, utilizou-se de um triângulopadrão, de acordo com metodologia proposta por RIPOLI (1996), com amostragens feitas ao acaso e em diferentes fileiras de plantio, tomando-se 20 amostras.

Em cada um dos 25 pontos de cada malha, foi realizada a amostragem para a determinação das perdas visíveis, utilizando-se de armação de 3,00 x 3,33 m. Todos os pontos foram estaqueados, e a amostragem seguiu a mesma seqüência de coleta de dados, ou seja, sempre à direita e à frente dos respectivos pontos, tomando-se todos os rizomas.

As perdas visíveis foram coletadas dentro do limite da armação de 3,00 x 3,33 m e, em seguida, foram separadas, pesadas e quantificadas, de acordo com os tipos (rebolo repicado, rebolo estilhaçado, pedaço fixo, pedaço solto, cana inteira, cana-ponta, estilhaço, toco e total) apresentados na Tabela 1.

Inicialmente, os dados foram analisados por meio da estatística descritiva para permitir a visualização geral do comportamento dos dados (VIEIRA et al., 2002), por meio do programa Minitab $^{\circledR}$. Foram calculados a média aritmética, a mediana, a amplitude, o desvio-padrão e os coeficientes de variação, de assimetria e de curtose. A averiguação da normalidade dos dados foi realizada pelo teste de Anderson-Darling.

Os métodos estatísticos utilizados para as determinações de perdas foram os histogramas, as cartas de controle por variáveis e o teste T. As cartas de controle foram feitas a partir dos limites inferior e superior de controle, elaboradas no programa Minitab ${ }^{\circledR}$. 
TABELA 1. Tipos de perdas amostradas. Types of losses sampled.

\begin{tabular}{lll}
\hline Tipo de Perdas & Especificação \\
Rebolo repicado & $\begin{array}{l}\text { Fração do colmo com o corte característico do } \\
\text { facão picador ou do corte de base, em ambas a } \\
\text { extremidades. }\end{array}$ \\
Rebolo estilhaçado & $\begin{array}{l}\text { Fração do colmo com pontas e/ou meio } \\
\text { dilacerados. }\end{array}$ \\
\hline Cana inteira & $\begin{array}{l}\text { Fração de cana com tamanho igual ou superior a } \\
\text { não do comprimento total. Esse colmo pode ou } \\
\text { nãoso ao solo pelas raízes. }\end{array}$ \\
\hline
\end{tabular}

Cana-ponta

Fração de colmo deixada no solo e agregada ao ponteiro.

Estilhaço

Fragmentos de cana dilacerados.
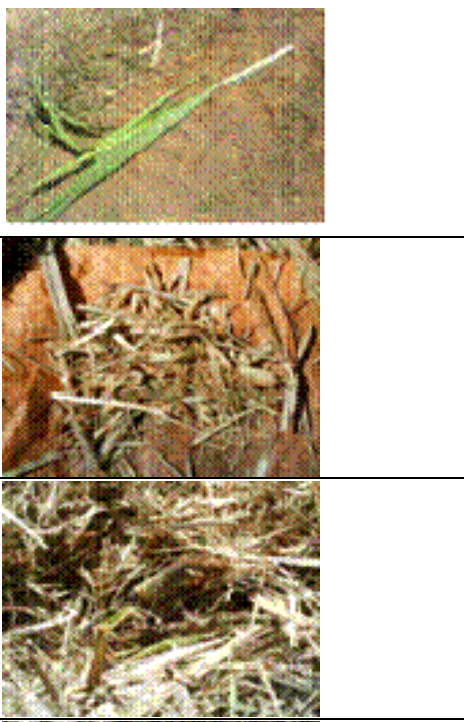

Toco

Fração do colmo cortada acima da superfície do solo, presa às raízes não-arrancadas, com comprimento menor ou igual a $0,2 \mathrm{~m}$

Pedaço Fixo

Segmento médio de cana (maior que $0,20 \mathrm{~m}$ ), que deve estar necessariamente preso ao solo.

Todas as variações visíveis de colmos sem as características que definam tocos, colmos Pedaço Solto inteiros, rebolos, lascas e cana-ponta e que, portanto, não se encaixam em nenhuma das definições anteriormente citadas.

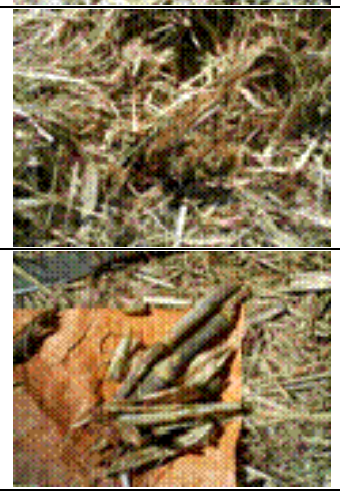

Para estimar as linhas médias e os limites dos gráficos de controle, foram usadas as seguintes equações (TRINDADE et al., 2000):

$$
\begin{aligned}
& \bar{X}=\mu \\
& \mathrm{UB}=\mu+3 \frac{\sigma}{\mathrm{c}_{2} \sqrt{\mathrm{n}}} \\
& \mathrm{LB}=\mu+3 \frac{\sigma}{\mathrm{c}_{2} \sqrt{\mathrm{n}}}
\end{aligned}
$$

em que, 
$\overline{\mathrm{X}}$ - linha média;

$\mu$ - média das médias dos subgrupos;

UB - limite superior de controle;

$\sigma$ - desvio-padrão;

$c_{2}$ - fator de ajuste à distribuição normal, tabelado em função de n;

$\mathrm{n}$ - tamanho da amostra, e

LB - limite inferior de controle.

Quando o cálculo do LB resultou em valores negativos, considerou-se o mesmo com valor nulo $(\mathrm{LB}=0)$.

\section{RESULTADOS E DISCUSSÃO}

Foram avaliados 313 e 245 colmos para o $3^{-0}$ e $4^{0}$ cortes, respectivamente. Desses totais, o canavial de $3^{\circ}$ corte estava com $46 \%$ dos colmos eretos, enquanto, no $4^{\circ}$ corte, $56 \%$. De acordo com a caracterização proposta por RIPOLI (1996), as áreas amostradas apresentaram-se eretas em ambos os cortes, apresentando $46 \%$ e $56 \%$ de colmos eretos para o $3^{\mathrm{o}}$ e $4^{\mathrm{o}}$ cortes, respectivamente. $\mathrm{O}$ percentual de colmos acamados e deitados foi de $34 \%$ e $20 \%$ para o $3^{\circ}$ corte, e de $27 \%$ e $17 \%$ para o $4^{\mathrm{O}}$ corte. Esses resultados indicam que os canaviais se encontravam eretos, porém com baixa uniformidade, pois resultados de RIPOLI (2001a) indicam valores de até $84 \%$ dos colmos eretos.

Assim, RIPOLI \& RIPOLI (2004) afirmam que as máquinas poderão desenvolver maior velocidade, oferecendo maior quantidade de matéria-prima de melhor qualidade com menores perdas visíveis. Dessa forma, a baixa uniformidade encontrada pode ser fator preponderante para o aumento das perdas durante a colheita, uma vez que pode dificultar a ação do corte de base da colhedora quando da presença de colmos acamados.

Na Tabela 2, são apresentadas as médias para as perdas visíveis de cana-de-açúcar nos talhões de $3^{-}$e $4^{-}$cortes, enquanto, na Tabela 3 , são apresentados os demais parâmetros da estatística descritiva das perdas na colheita.

TABELA 2. Análise estatística para as perdas na colheita de cana-de-açúcar no $3^{\circ}$ e $4^{\circ}$ cortes. Statistical analysis for the sugar cane harvest losses in $3^{\text {rd }}$ and $4^{\text {th }}$ cuts.

\begin{tabular}{|c|c|c|c|c|c|}
\hline \multirow{3}{*}{$\frac{\text { Parâmetro }}{\text { Rebolo repicado }}$} & \multirow{3}{*}{$\begin{array}{c}\text { Corte } \\
3^{\underline{o}} \\
4^{\underline{o}} \\
\end{array}$} & \multicolumn{3}{|c|}{ Perdas $\left(\mathrm{kg} \mathrm{ha}^{-1}\right)$} & \multirow{2}{*}{$\begin{array}{l}\text { Coeficiente de } \\
\text { Variação (\%) }\end{array}$} \\
\hline & & Média (corte)* & \multicolumn{2}{|c|}{ Média (tipo) } & \\
\hline & & $\begin{array}{l}248 \mathrm{a} \\
516 \mathrm{a}\end{array}$ & 382 & $\mathrm{BC}$ & 247,2 \\
\hline Rebolo estilhaçado & $\begin{array}{l}3^{\mathrm{o}} \\
4^{-}\end{array}$ & $\begin{array}{l}904 \mathrm{a} \\
672 \mathrm{~b}\end{array}$ & 788 & $\mathrm{AB}$ & 27,9 \\
\hline Pedaço fixo & $\begin{array}{l}3^{\mathrm{o}} \\
4^{-}\end{array}$ & $\begin{array}{r}8 \mathrm{~b} \\
212 \mathrm{a}\end{array}$ & 110 & $\mathrm{C}$ & 222,9 \\
\hline Pedaço solto & $\begin{array}{l}3^{\mathrm{o}} \\
4^{-}\end{array}$ & $\begin{array}{r}1.128 \mathrm{a} \\
992 \mathrm{a}\end{array}$ & 1.060 & A & 27,0 \\
\hline Cana inteira & $\begin{array}{l}3^{-} \\
4^{-}\end{array}$ & $\begin{array}{l}100 \mathrm{a} \\
300 \mathrm{a}\end{array}$ & 200 & $\mathrm{C}$ & 274,8 \\
\hline Cana-ponta & $\begin{array}{l}3^{\mathrm{o}} \\
4^{-}\end{array}$ & $\begin{array}{r}0 \mathrm{a} \\
16 \mathrm{a} \\
\end{array}$ & 8 & $\mathrm{C}$ & 489,5 \\
\hline Estilhaço & $\begin{array}{l}3^{\mathrm{o}} \\
4^{\mathrm{o}}\end{array}$ & $\begin{array}{l}300 \mathrm{a} \\
232 \mathrm{a}\end{array}$ & 266 & $\mathrm{BC}$ & 59,3 \\
\hline Toco & $\begin{array}{l}3^{\mathrm{o}} \\
4^{\mathrm{o}}\end{array}$ & $\begin{array}{r}8 \mathrm{~b} \\
88 \mathrm{a}\end{array}$ & 48 & $\mathrm{C}$ & 209,4 \\
\hline Total & $\begin{array}{l}3^{-} \\
4^{-}\end{array}$ & $\begin{array}{l}2.696 \mathrm{a} \\
3.028 \mathrm{a}\end{array}$ & 2.862 & & 45,0 \\
\hline
\end{tabular}

Produtividade: $3^{\circ}$ corte: $62,3 \mathrm{Mg}^{-1}$

* Médias seguidas de mesma letra minúscula na coluna não diferem entre si, pelo teste de Tukey, a $5 \%$ de probabilidade, e comparam os anos de corte de cada variável. Médias seguidas por mesma letra maiúscula na coluna não diferem entre si, pelo teste de Tukey, a 5\% de probabilidade, e comparam os tipos de perdas, independentemente do ano de corte. 
Analisando-se os valores médios de cada variável (Tabela 2), observa-se que as perdas de pedaço solto foram estatisticamente maiores que as de pedaço fixo, cana inteira, cana-ponta e toco. Avaliando cada corte, verifica-se que as perdas de rebolo estilhaçado foram maiores no $3^{-0}$ corte, enquanto as perdas em pedaço fixo e toco foram maiores no $4^{\circ}$ corte. A maior ocorrência desse tipo de perdas pode ser explicada pela baixa uniformidade do porte do canavial, visto que há alta percentagem de colmos acamados e deitados, $34 \%$ e $20 \%$, respectivamente. Os demais tipos de perdas não apresentaram diferenças estatísticas em função do corte realizado, o que pode ser justificado pelos altíssimos valores dos coeficientes de variação encontrados. A produtividade média obtida nas áreas de $3^{-0}$ e $4^{0}$ cortes foi de $62,3 \mathrm{Mg} \mathrm{ha}^{-1}$ e $64,4 \mathrm{Mg} \mathrm{ha}^{-1}$, respectivamente.

Fica evidenciado na Tabela 2 que as maiores perdas ocorrem nos rebolos estilhaçados e pedaço solto, o que, segundo RIPOLI \& RIPOLI (2004), dá-se pela alta rotação de trabalho do exaustor primário. Provavelmente, essa anormalidade nas perdas em pedaço solto influenciou diretamente nas perdas totais nesse talhão. O nível médio de perdas para a colheita no talhão de $4^{\underline{0}}$ corte $\left[3.028 \mathrm{~kg} \mathrm{ha}^{-1}(4,70 \%)\right]$ e no talhão de $3^{\circ}$ corte $\left[2.696 \mathrm{~kg} \mathrm{ha}^{-1}(4,33 \%)\right]$ manteve-se dentro dos padrões preconizados por NEVES et al. (2003) e NEVES et al. (2004), como aceitáveis para a cultura da cana-de-açúcar.

Observando-se a Tabela 3, constata-se que existe distribuição anormal em todas as variáveis de perdas, exceto em relação às perdas em pedaço solto e totais para o $3^{0}$ corte.

TABELA 3. Estatísticas descritivas das variáveis das perdas de cana-de-açúcar nos talhões de $3^{\circ}$ e $4^{\circ}$ cortes: rebolo repicado (RR), rebolo estilhaçado (RE), pedaço fixo (PF), pedaço solto (PS), cana inteira (CI), cana-ponta (CP), estilhaço (E), toco (TO) e perdas totais (PT). Descriptive statistics of the variables of the loss of sugar cane in the stands of $3^{\text {rd }}$ and $4^{\text {th }}$ cuts: diced stalks (RR), splinted stalks (RE), fixed piece (PF), untied piece (PS), whole cane (CI), cane top (CP), splints (E), stubbles (TO) and total losses (PT).

\begin{tabular}{|c|c|c|c|c|c|c|c|c|}
\hline \multirow{3}{*}{ Perdas } & \multicolumn{8}{|c|}{ Medidas Estatísticas Descritivas (\%) } \\
\hline & \multirow{2}{*}{ Média } & \multirow{2}{*}{ Mediana } & \multirow{2}{*}{ Amplitude } & \multirow{2}{*}{$\begin{array}{l}\text { Desvio- } \\
\text { Padrão }\end{array}$} & \multicolumn{2}{|c|}{ Coeficiente } & \multirow{2}{*}{ Teste $^{*}$} & \multirow{2}{*}{$\mathrm{P}$} \\
\hline & & & & & $\mathrm{Ck}$ & Cs & & \\
\hline $\mathrm{RR}\left(3^{\mathrm{O}}\right)$ & 0,40 & 0,20 & 1,60 & 0,48 & 1,19 & 1,28 & A & 0,000 \\
\hline $\mathrm{RR}\left(4^{\mathrm{o}}\right)$ & 0,81 & 0,50 & 10,30 & 2,03 & 22,26 & 4,62 & A & 0,000 \\
\hline $\mathrm{RE}\left(3^{\mathrm{o}}\right)$ & 1,40 & 1,40 & 1,20 & 0,31 & $-0,13$ & $-0,63$ & A & 0,006 \\
\hline $\operatorname{RE}\left(4^{\circ}\right)$ & 1,04 & 0,90 & 1,60 & 0,37 & 1,16 & 1,05 & A & 0,022 \\
\hline $\mathrm{PF}\left(3^{\underline{o}}\right)$ & 0,01 & 0,00 & 0,30 & 0,06 & 25,00 & 5,00 & A & 0,000 \\
\hline $\operatorname{PF}\left(4^{\underline{0}}\right)$ & 0,34 & 0,00 & 1,60 & 0,55 & 1,16 & 1,53 & A & 0,000 \\
\hline PS $\left(3^{0}\right)$ & 1,80 & 1,80 & 1,30 & 0,31 & $-0,04$ & 0,013 & $\mathrm{~N}$ & 0,084 \\
\hline $\operatorname{PS}\left(4^{\circ}\right)$ & 1,55 & 1,60 & 2,30 & 0,56 & 1,10 & 0,73 & A & 0,039 \\
\hline $\mathrm{CI}\left(3^{\mathrm{o}}\right)$ & 0,16 & 0,00 & 1,60 & 0,46 & 6,91 & 2,82 & A & 0,000 \\
\hline $\mathrm{CI}\left(4^{\mathrm{o}}\right)$ & 0,47 & 0,00 & 4,70 & 1,13 & 8,75 & 2,93 & A & 0,000 \\
\hline $\mathrm{CP}\left(3^{\underline{o}}\right)$ & 0,00 & 0,00 & 0,00 & 0,00 & 0,00 & 0,00 & $\mathrm{~A}$ & 0,000 \\
\hline $\mathrm{CP}\left(4^{\underline{0}}\right)$ & 0,02 & 0,00 & 0,30 & 0,08 & 9,64 & 3,30 & A & 0,000 \\
\hline $\operatorname{ES}\left(3^{\underline{o}}\right)$ & 0,50 & 0,50 & 1,10 & 0,31 & 0,80 & 1,24 & A & 0,000 \\
\hline $\operatorname{ES}\left(4^{\circ}\right)$ & 0,37 & 0,30 & 0,60 & 0,16 & 0,59 & 1,10 & A & 0,000 \\
\hline $\mathrm{TO}\left(3^{\mathrm{o}}\right)$ & 0,01 & 0,00 & 0,30 & 0,06 & 25,00 & 5,00 & $\mathrm{~A}$ & 0,000 \\
\hline TO $\left(4^{\mathrm{o}}\right)$ & 0,14 & 0,00 & 0,80 & 0,22 & 2,11 & 1,56 & A & 0,000 \\
\hline $\mathrm{PT}\left(3^{\underline{o}}\right)$ & 4,33 & 4,50 & 4,20 & 1,13 & $-0,92$ & $-0,08$ & $\mathrm{~N}$ & 0,379 \\
\hline $\mathrm{PT}\left(4^{\mathrm{o}}\right)$ & 4,70 & 3,70 & 11,20 & 2,62 & 5,02 & 2,10 & A & 0,000 \\
\hline
\end{tabular}

Ck: coeficiente de curtose; Cs: coeficiente de assimetria; ${ }^{*} \mathrm{~N}$ : distribuição normal pelo teste de Anderson-Darling; A: distribuição assimétrica; P: valor de probabilidade, pelo teste de Anderson-Darling. 


\section{Controle estatístico de processo para perdas visíveis}

\section{Perdas em rebolo repicado}

Na Figura 1, são apresentadas as cartas de controle das médias para a variável perdas em rebolo repicado, nos talhões de $3^{\underline{0}}$ e $4^{\underline{0}}$ cortes. Observa-se que, em ambas as situações, o processo encontra-se fora de controle. Há, porém, mais pontos dentro dos limites de controle para o $4^{\mathrm{O}}$ corte, mostrando que a operação de colheita nesse talhão ocorreu de forma mais próxima do controle de qualidade do que a colheita da cana-de-açúcar no $3^{0}$ corte.

Considerando-se que perdas em rebolos repicados ocorrem principalmente devido ao erro humano, pode-se perceber que, provavelmente, ao conduzir a colhedora ou o transbordo, houve falta de atenção do operador, provocando o excesso de carregamento ou, então, a descarga fora do transbordo. É possível que a maior percentagem de perdas no $4^{\underline{0}}$ corte tenha ocorrido durante a noite, visto que a colheita desse talhão se iniciou nesse período, quando a visibilidade de descarga no transbordo é deficiente.
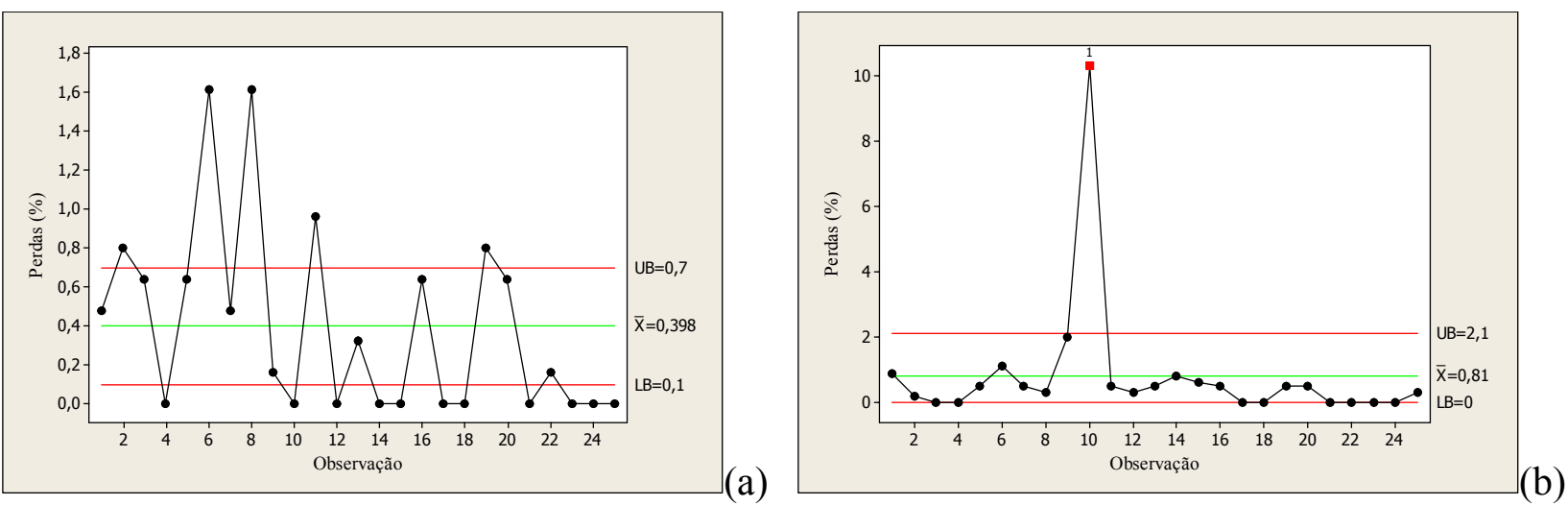

FIGURA 1. Carta de controle para as perdas de rebolo repicado: a) $3^{\underline{0}}$ corte e b) $4^{\underline{0}}$ corte. Control letter for the losses of diced stalks: a) $3^{\text {rd }}$ cut and b) $4^{\text {th }}$ cut.

\section{Perdas em rebolo estilhaçado}

As cartas de controle para a variável perdas em rebolo estilhaçado (Figura 2) indicam que o processo também se encontra fora de controle, visto que há pontos que saem dos limites nas duas cartas. Como essas perdas estão relacionadas com o exaustor primário, quando a velocidade desse está muito elevada, o rebolo repicado será sugado junto com a terra e a palha, sendo lançado fora dos transbordos como matéria estranha. Ao passar pelo ventilador, os rebolos são atingidos pelas pás, sendo, então, estilhaçados. De acordo com as cartas de controle, nota-se que há mais pontos entre os limites de controle para o $3^{0}$ corte. As perdas médias do rebolo estilhaçado ficaram próximas a $1,0 \%$ e a $1,5 \%$, no $4^{\circ}$ e no $3^{\circ}$ cortes, respectivamente; segundo RIPOLI (2001c), essa perda é classificada como rebolos rachados, e em suas pesquisas ocorrem na ordem de $3,6 \%$, sendo esse dado obtido por meio de vários ensaios de diversas colhedoras.
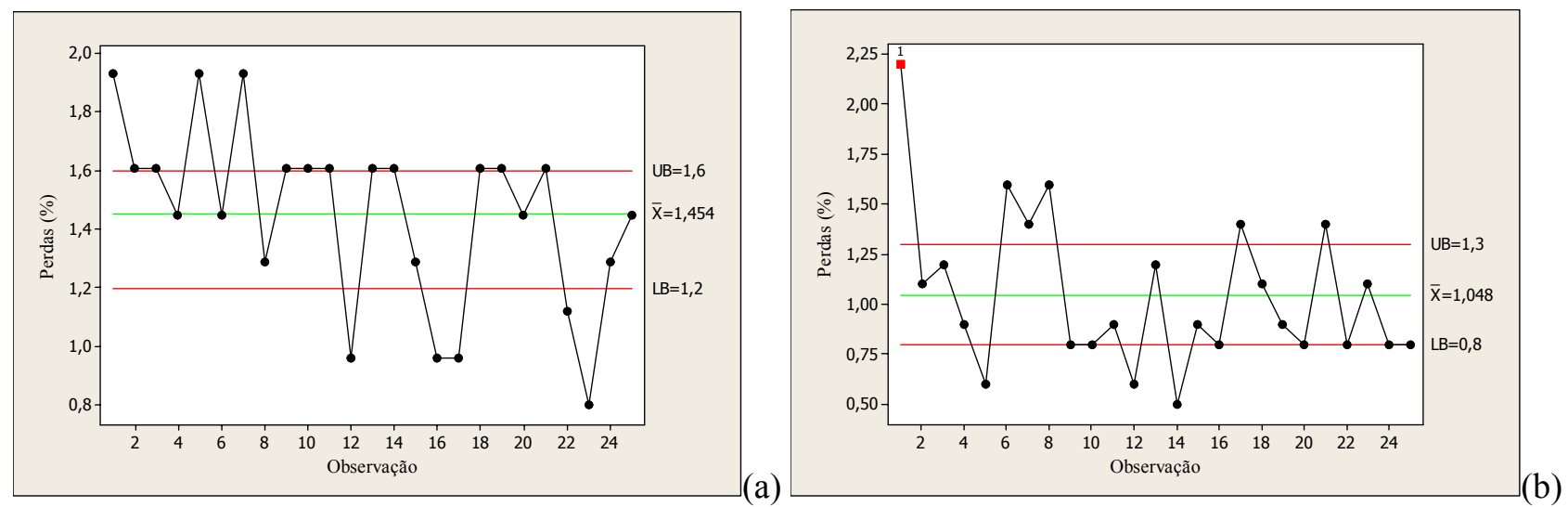

FIGURA 2. Carta de controle para as perdas de rebolo estilhaçado: a) $3^{\circ}$ corte e b) $4^{\circ}$ corte. Control letter for the losses of splinted stalks: a) $3^{\text {rd }}$ cut and b) $4^{\text {th }}$ cut. 


\section{Perdas em pedaço fixo}

As perdas relacionadas a pedaços fixos (pedaços de aproximadamente $5 \mathrm{~cm}$ ) indicam que o corte foi efetuado acima do desejado, devido a, normalmente, terrenos acidentados ou mudança na altura de corte durante o processo. Na Figura 3, pode-se observar que houve falta de controle em ambos os talhões, principalmente no de $4^{\underline{0}}$ corte, onde houve mais ocorrência desse tipo de perda. No $3^{\underline{0}}$ corte, ocorreu um único ponto, o que pode representar um "outlier", ou seja, pode ser considerado como erro de amostragens, conforme PORTELA (2005). Outro fator que pode justificar as perdas por pedaço fixo é a colheita ter sido realizada no período noturno.
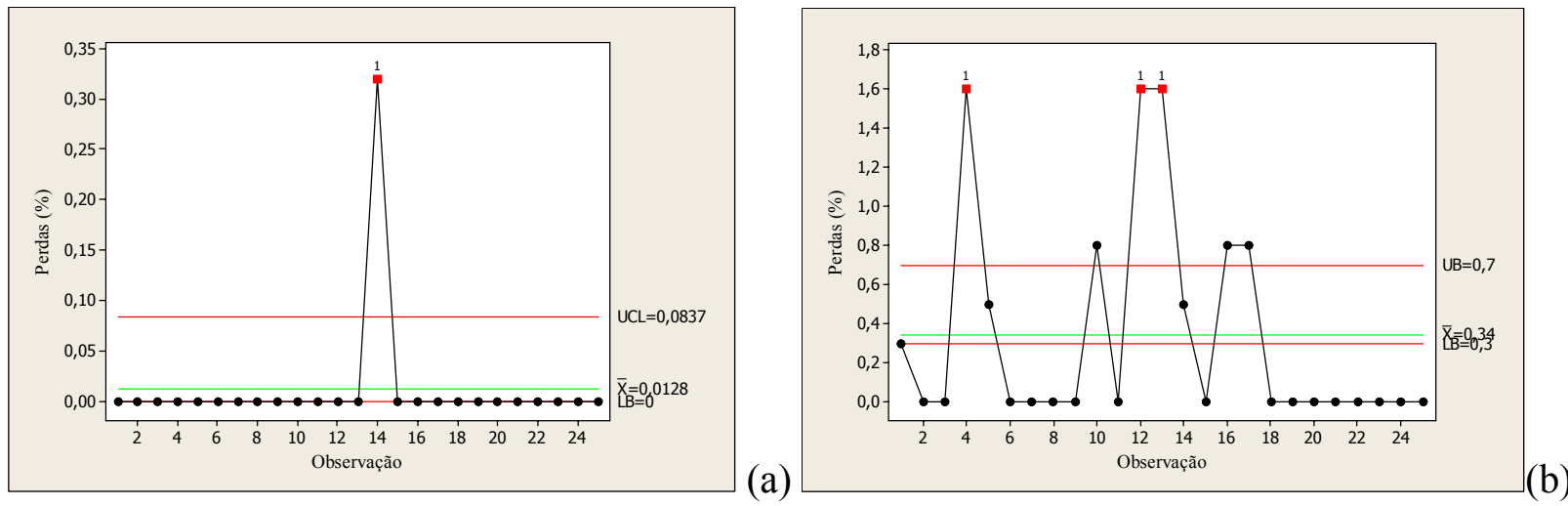

FIGURA 3. Carta de controle para as perdas de pedaço fixo: a) $3^{\circ}$ corte e b) $4^{\circ}$ corte. Control letter for the losses of fixed piece: a) $3^{\text {rd }}$ cut and b) $4^{\text {th }}$ cut.

\section{Perdas em pedaço solto}

Encontram-se representadas na Figura 4 as cartas de controle para as perdas em pedaço solto, relativas ao $3^{\underline{0}}$ e $4^{\underline{0}}$ cortes. De acordo com a análise das cartas, o processo em questão encontra-se fora de controle. Esse tipo de perda ocorre da mesma forma que o rebolo estilhaçado, porém suas dimensões diferenciam-no do rebolo estilhaçado devido ao corte no picador de colmos que, em geral, são maiores, apresentando até dois entrenós. A incidência maior de pontos entre os limites na carta do $4^{\underline{0}}$ corte mostra maior controle na colheita desse talhão. Para RIPOLI (2001c), as perdas de rebolos inteiros ou pedaços soltos foram de $1,2 \%$, abaixo do resultado deste experimento que foi, em média, de $1,8 \%$ para $3^{-}$corte e $1,5 \%$ para $4^{\circ}$ corte.
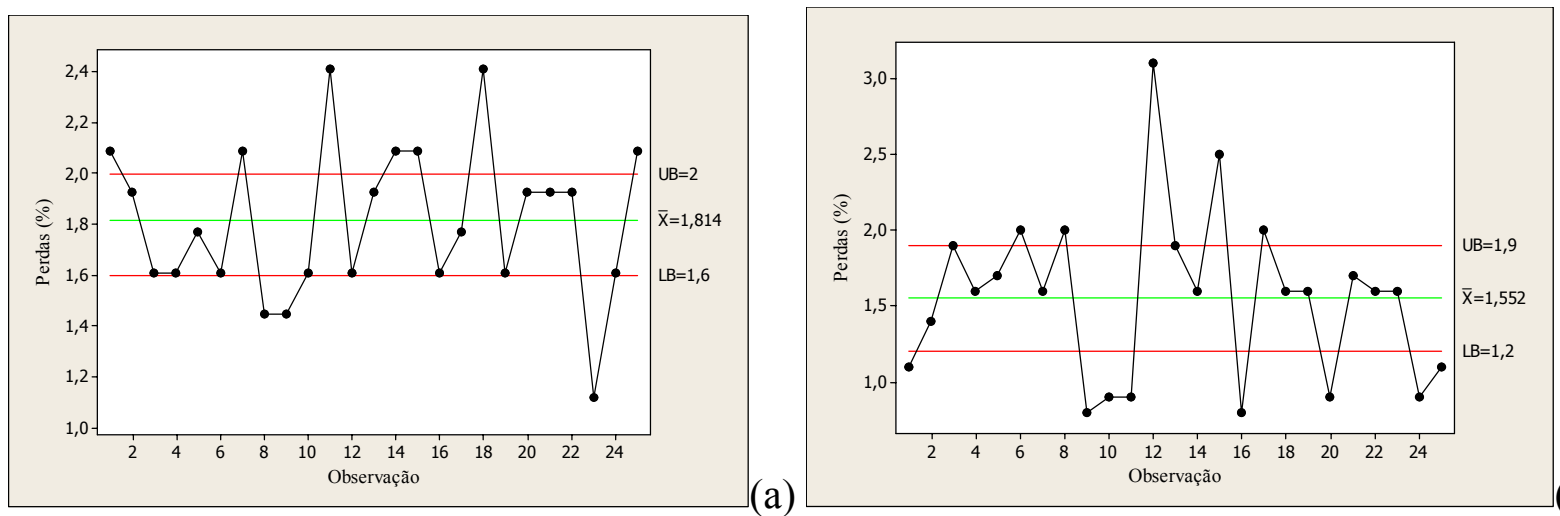

FIGURA 4. Carta de controle para as perdas de pedaço solto: a) $3^{\underline{o}}$ corte e b) $4^{\underline{o}}$ corte. Control letter for the losses of untied piece: a) $3^{\text {rd }}$ cut and b) $4^{\text {th }}$ cut.

\section{Perdas em cana inteira}

A perda em cana inteira pode estar relacionada ao acamamento ou à presença de plantas deitadas; quando essas ocorrem em condições extremas, torna ineficiente o trabalho do levantador de colmo da colhedora. As cartas de controle para essas perdas no $3^{\underline{0}}$ e no $4^{\underline{0}}$ cortes estão representadas na Figura 5, na qual se observa a falta de controle do processo, embora exista pouca 
incidência dessas perdas. O fato é que o canavial nos talhões amostrados se apresentou com porte ereto, o que facilitou o trabalho da colhedora.
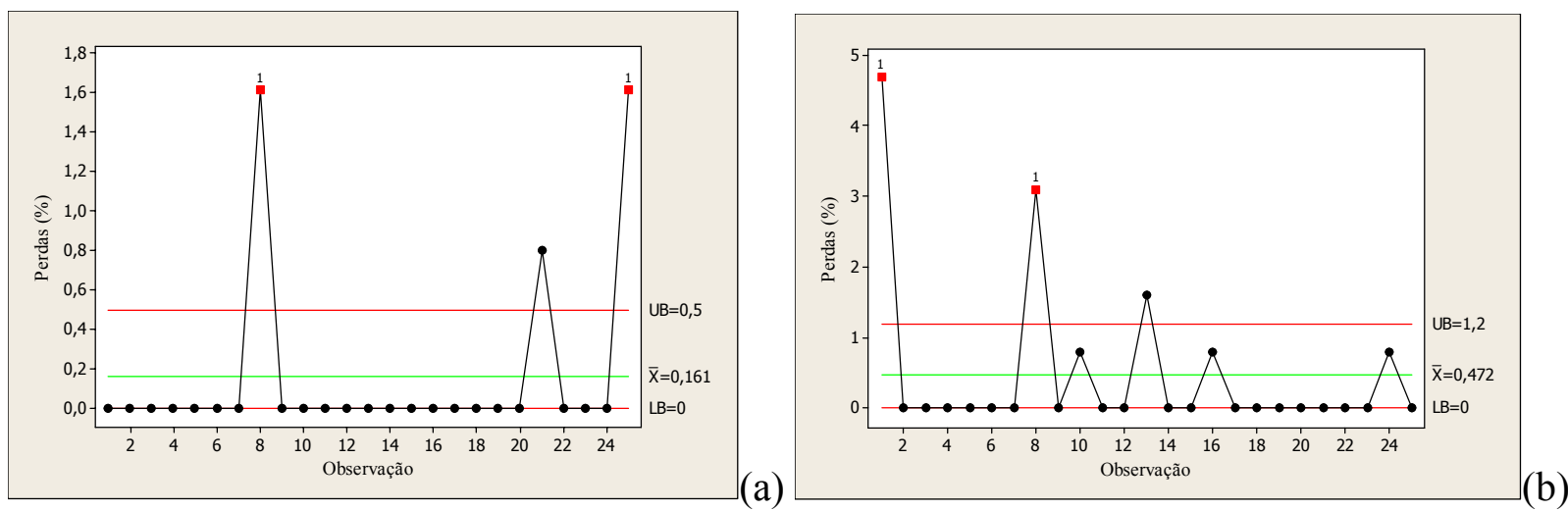

FIGURA 5. Carta de controle para as perdas de cana inteira: a) $3^{\circ}$ corte e b) $4^{\circ}$ corte. Control letter for the losses of whole cane: a) $3^{\text {rd }}$ cut and b) $4^{\text {th }}$ cut.

\section{Perdas em cana-ponta}

Observam-se, na Figura 6 , apenas as perdas em cana-ponta relativa ao $4^{\circ}$ corte, uma vez que, no $3^{-}$corte, esse tipo de perdas não foi encontrado. No talhão de $3^{-}$corte, a colhedora trabalhou com o cortador de ponteiros desligado, sistema que é acionado quando há a necessidade de desponte. Geralmente, quando a altura das plantas ultrapassa o dispositivo, não há a necessidade nem a conveniência de fazer o desponte. Notam-se pontos que confirmam a existência de canaponta apenas no $4^{\underline{0}}$ corte, talhão no qual o despontador se encontrava ligado, propiciando o corte das pontas da cana-de-açúcar. As perdas foram baixas, o que pode ser considerado devido à correta regulagem da altura do mecanismo de desponte.

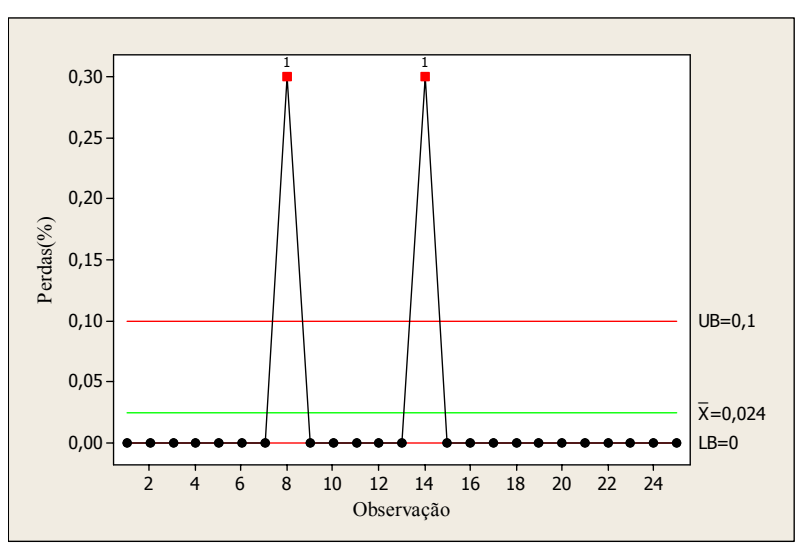

FIGURA 6. Carta de controle para as perdas de cana-ponta para o $4^{-}$corte. Control letter for the losses of cane top: a) $3^{\text {rd }}$ cut and b) $4^{\text {th }}$ cut.

\section{Perdas em estilhaço}

Nas cartas de controle representadas na Figura 7, podem ser observadas as perdas em estilhaço relativas aos talhões de $3^{\underline{0}}$ e $4^{\underline{0}}$ cortes. O corte da planta nos picadores em frações menores que as desejadas, aliados à velocidade dos ventiladores de exaustão dilaceram os fragmentos do colmo. Apesar de ambos os talhões apresentarem-se fora de controle, é possível identificar que houve maior uniformidade do processo no $4^{0}$ corte pelo maior número de pontos dentro dos limites inferior e superior de controle. 

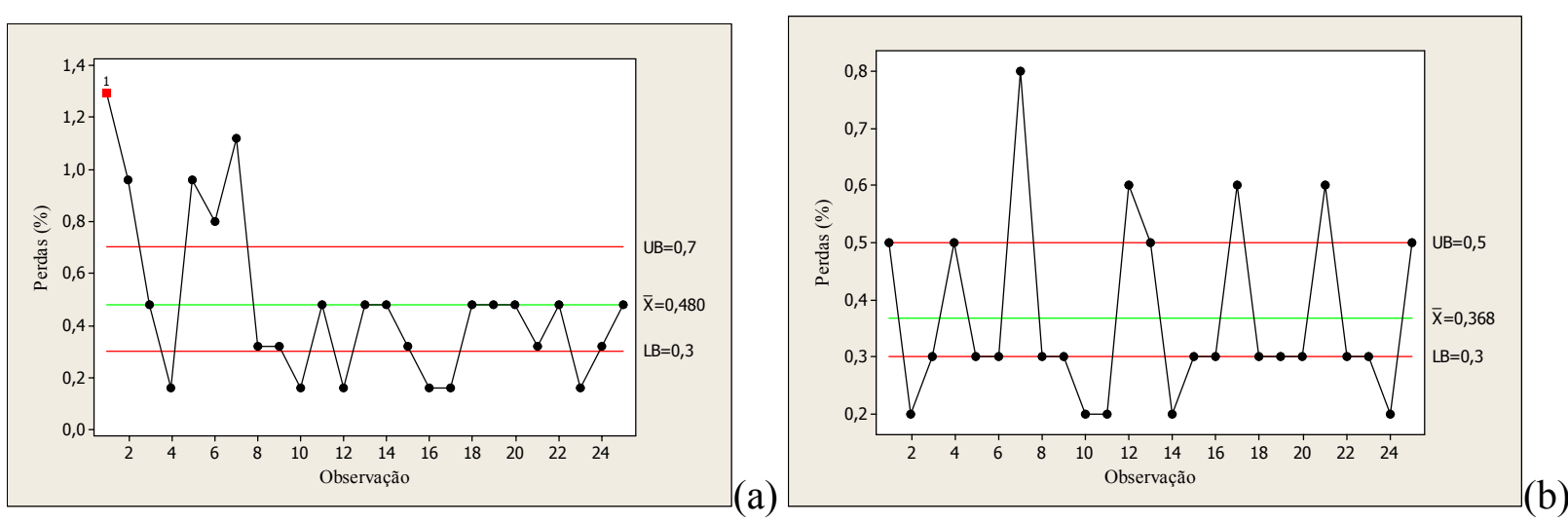

FIGURA 7. Carta de controle para as perdas de estilhaço: a) $3^{-}$corte e b) $4^{\underline{0}}$ corte. Control letter for the losses of splints: a) $3^{\text {rd }}$ cut and b) $4^{\text {th }}$ cut.

\section{Perdas em toco}

Observa-se nas cartas de controle, correspondentes aos talhões de $3^{\underline{0}}$ e $4^{\underline{o}}$ cortes (Figura 8), que o índice de perdas em tocos foi baixo. Apesar de essas cartas indicarem o processo fora de controle, nota-se que há semelhança com as perdas relacionadas ao pedaço fixo, o que é justificado pelo fato de ambos os tipos de perdas serem causadas pelos mesmos motivos.
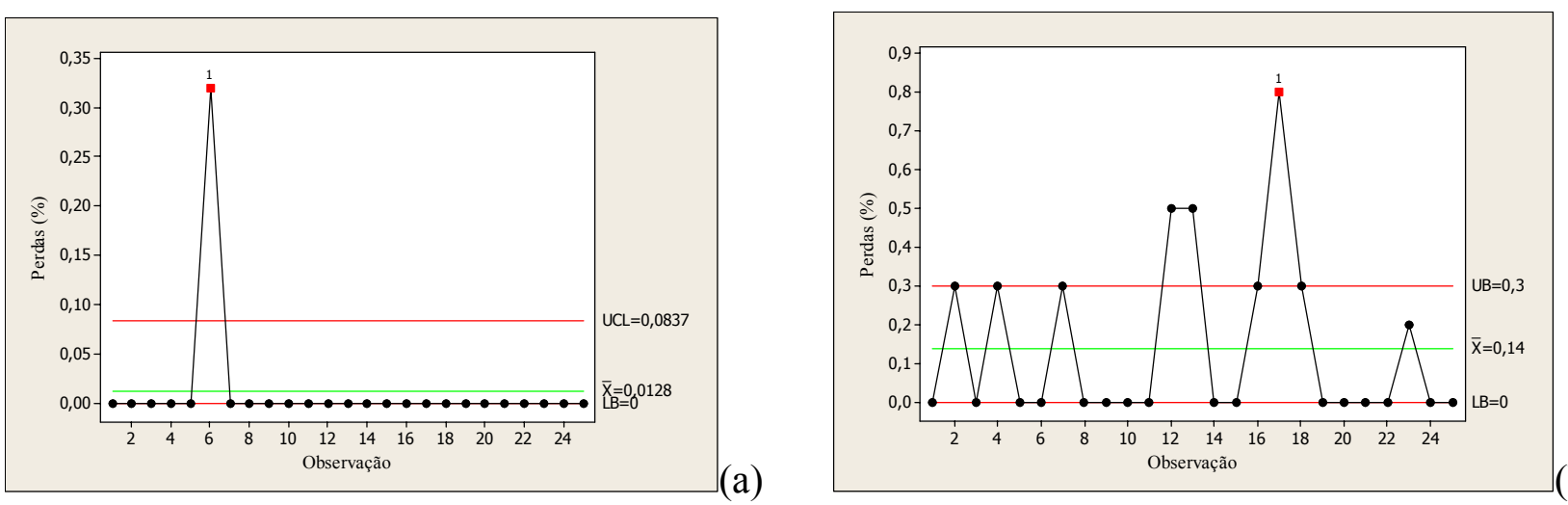

FIGURA 8. Carta de controle para as perdas de toco: a) $3^{-0}$ corte e b) $4^{\underline{0}}$ corte. Control letter for the losses of stubbles: a) $3^{\text {rd }}$ cut and b) $4^{\text {th }}$ cut.

\section{Perdas totais}

Nas cartas de controle para perdas totais (Figura 9), nota-se que, considerando-se as perdas como indicadores de qualidade, o processo da colheita mecanizada está fora de controle em ambos os cortes. Entretanto, a quantidade de pontos dentro dos limites de controle no $4^{\underline{0}}$ corte foi maior do que no $3^{\mathrm{o}}$ corte. RIPOLI (2001a), avaliando a colheita mecanizada da cana-de-açúcar, com a variedade RB83-5089, verificou perdas de 8,8\% na colhedora Brasoft. Na mesma condição, RIPOLI (2001b) encontrou perdas de 5,6\% para a colhedora Claas Ventor, resultados esses bem próximos deste experimento. Da mesma forma, NEVES et al. (2004) verificaram na colheita mecanizada da variedade RB72-454, de terceiro corte, colhida com uma colhedora Cameco CHT 2500 , perdas totais de $5,68 \%$. 

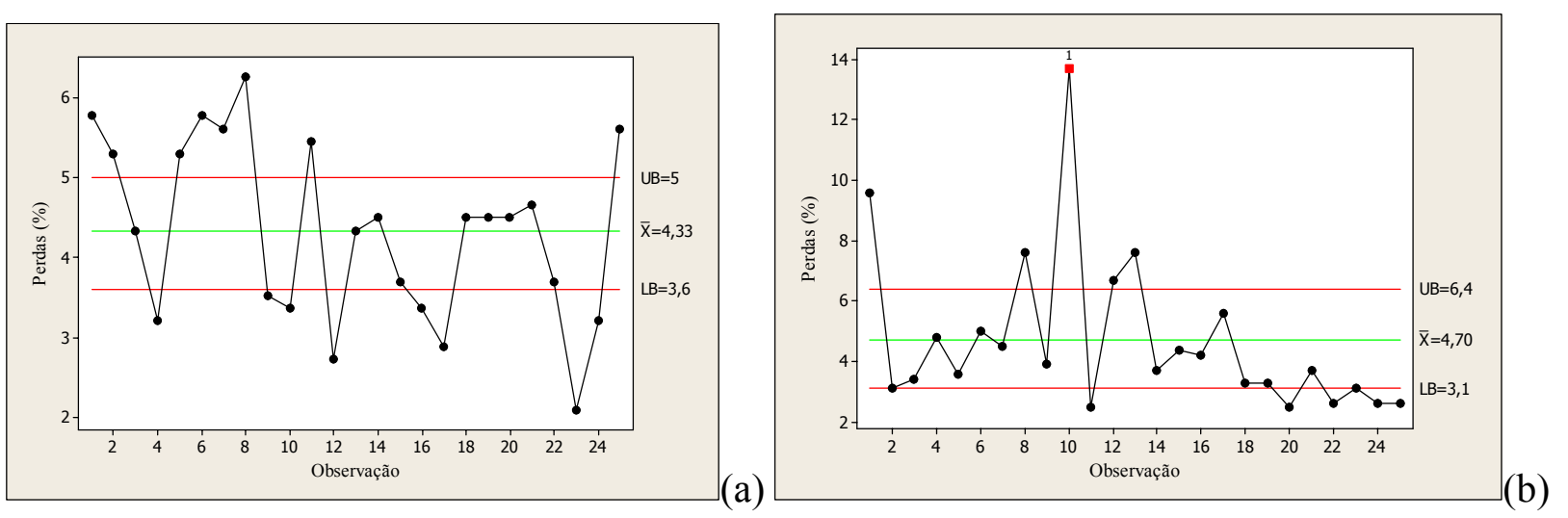

FIGURA 9. Carta de controle para as perdas totais: a) $3^{-0}$ corte e b) $4^{\underline{0}}$ corte. Control letter for the total losses: a) $3^{\text {rd }}$ cut and b) $4^{\text {th }}$ cut.

\section{CONCLUSÕES}

As perdas de pedaço solto foram superiores às perdas de pedaço fixo, cana inteira, cana-ponta e toco na média do $3^{\mathrm{o}}$ e $4^{\mathrm{o}}$ cortes.

As perdas em rebolo estilhaçado foram menores no $4^{0}$ corte em relação ao $3^{0}$ corte, enquanto as perdas em pedaço fixo e toco foram menores no $3^{-}$corte, comparadas ao $4^{-}$corte.

A variável pedaço solto apresentou a maior percentagem de perdas em relação às demais variáveis.

Os indicadores perdas na colheita demonstraram que a colheita mecanizada não se encontra sob controle estatístico de processo, ou seja, fora do padrão de qualidade.

\section{REFERÊNCIAS}

BONILLA, J.A. Qualidade total na agricultura: fundamentos e aplicações. Belo Horizonte: Centro de Estudos da Qualidade Total na Agricultura, 1995. 344 p.

CARVALHO FILHO, S.M. Colheita mecanizada: desempenho operacional e econômico em cana sem queima prévia. 2000. 108 f. Dissertação (Mestrado em Máquinas Agrícolas) - Escola Superior de Agricultura “Luiz de Queiroz", Universidade de São Paulo, Piracicaba, 2000.

DODSON, M.S. Avaliação da influência de indicadores de qualidade no custo operacional de um sistema de produção de milho Zea mays (L.): estudo de caso de semeadura. 1998. $80 \mathrm{f}$. Monografia (Trabalho de Graduação em Agronomia) - Universidade Estadual Paulista, Faculdade de Ciências Agrárias e Veterinárias, Jaboticabal, 1998.

FERNANDES, R.A.T.; MILAN, M.; PECHE FILHO, A. Gerenciamento da qualidade em operações mecanizadas de um sistema de produção de cana-de-açúcar. Engenharia Agrícola, Jaboticabal, v.20, n.3, p.215-20, 2000.

GARCIA, A.P.; ALBIERO, D.; CAPPELLI, N.L.; MACIEL, A.J.S.; UMEZU, C.K. Avaliação das perdas na colheita de cana-de-açúcar e demanda de potência de uma colhedora de cana inteira. In: CONGRESSO BRASILEIRO DE ENGENHARIA AGRÍCOLA, 35., 2006, João Pessoa. Anais... Jaboticabal: Associação Brasileira de Engenharia Agrícola, 2006. 1 CD-ROM.

MAGALHÃES, P.S.G.; MILAN, M.; MOLIN, J.P.; SOUZA, Z.M.; VOLPATO, C.E.; SIMÕES, J. Colheita de cana-de-açúcar e palha para a produção de Etanol. In: WORKSHOP - COLHEITA, TRANSPORTE E RECUPERAÇÃO DE PALHA, 2., 2006, Campinas: Universidade Estadual de Campinas, 2006. 19 p.

MIGNOTI, S.A.; FIDELIS, M.T. Aplicando a geoestatística no controle estatístico de processo. Revista Produto \& Produção, Porto Alegre, v.5, n.2, p.55-70, 2001. 
MILAN, M.; FERNANDES, R.A.T. Qualidade das operações de preparo de solo por controle estatístico de processo. Scientia Agrícola, Piracicaba, v.59, n.2, p.261-6, 2002.

NEVES, J.L.M.; MAGALHÃES, P.S.G.; MORAES, E.E.; ARAUJO, F. Avaliação de perdas invisíveis de cana-de-açúcar nos sistemas da colhedora de cana picada. Engenharia Agrícola, Jaboticabal, v.23, n.3, p.539-46, 2003.

NEVES, J.L.M.; MAGALHAES, P.S.G.; OTA, W.M. Sistema de monitoramento de perdas visíveis de cana-de-açúcar em colhedora de cana picada. Engenharia Agrícola, Jaboticabal, v.24, n.3, p.764-70, 2004.

PORTELLA, L.M. Parâmetros físicos do solo como base para a agricultura de precisão. 2005. 69 f. Monografia (Trabalho de Graduação em Agronomia) - Universidade Estadual Paulista, Faculdade de Ciências Agrárias e Veterinárias, Jaboticabal, 2005.

RIPOLI, T.C.C. Ensaio \& certificação de máquinas para colheita de cana-de-açúcar. In: MIALHE, L.G. Máquinas agrícolas: ensaios \& certificação. Piracicaba: Fundação de Estudos "Luiz de Queiroz", 1996. cap.13, p.635-73.

RIPOLI, T.C.C.; NERY, M.S.; de LEÓN, M.J.; PIEDADE, S.M.S. Desempenho operacional de uma colhedora em cana crua em função da velocidade de avanço. Engenharia Agrícola, Jaboticabal, v.19, n.2, p.199-207, 1999.

RIPOLI, T.C.C. Algumas considerações sobre palhiço como fonte de energia. In: SEMINÁRIO INTERNACIONAL CANA \& ENERGIA, 2001, Ribeirão Preto. Anais... Ribeirão Preto: INEE/ IDEA, 2001a. 1 CD-ROM.

RIPOLI, T.C.C. Algumas considerações sobre palhiço. In: SEMINÁRIO INTERNACIONAL CANA \& ENERGIA, 2001, Ribeirão Preto. Anais... Ribeirão Preto: INEE/IDEA, 2001b. 1 CDROM.

RIPOLI, T.C.C. Colhedora, colhedeira, colheitadora, colheitadeira ... que confusão! IDEA News, Ribeirão Preto, v.2, n.6, p.6, 2001c.

RIPOLI, T.C.C.; RIPOLI, M.L.C. Biomassa de cana-de-açúcar: colheita, energia e ambiente. Piracicaba: Ed. Barros \& Marques Editoração Eletrônica, 2004. 302 p.

TRINDADE, C.; REZENDE, J.L.P.; JACOVINE, L.A.G.; SARTORIO, M.L. Ferramentas da qualidade: aplicação na atividade florestal. Viçosa: Universidade Federal de Viçosa, 2000. 124 p.

UNB. Portal da Universidade de Brasília. Disponível em:

http://www.unb.br/ig/glossario/verbete/coordenadas_utm.htm. Acesso em: 6 dez. 2006.

VIEIRA, S.R.; MILLETE, J.; TOPP, G.C.; REYNOLDS, W.D. Handbook for geoestatical analysis of variability in soil and climate data. In: ALVAREZ V.V.H.; SCHAEFER, C.E.G.R.; BARROS, N.F.; MELLO, J.W.V.; COSTA, L.M. (Ed.) Tópicos em ciência do solo. Viçosa: Sociedade Brasileira de Ciência do Solo, 2002. v.2, p.1-45.

VOLPATO, C.E.S.; BRAUNBECK, O.A.; OLIVEIRA, C.A.A. Desenvolvimento e avaliação de um protótipo de cortador de base para colhedoras de cana-de-açúcar. Revista Brasileira de Engenharia Agrícola e Ambiental, Campina Grande, v.6, n.2, p.345-8, 2002. 